\title{
The Phase Diagram of the Quantum Curie-Weiss Model
}

\author{
Lincoln Chayes • Nicholas Crawford • Dmitry Ioffe • \\ Anna Levit
}

Received: 8 April 2008 / Accepted: 30 July 2008 / Published online: 29 August 2008

(C) Springer Science+Business Media, LLC 2008

\begin{abstract}
This paper studies a generalization of the Curie-Weiss model (the Ising model on a complete graph) to quantum mechanics. Using a natural probabilistic representation of this model, we give a complete picture of the phase diagram of the model in the parameters of inverse temperature and transverse field strength. Further analysis computes the critical exponent for the vanishing of the order parameter in the approach to the critical curve and gives useful stability properties for a variational problem associated with the representation.
\end{abstract}

Keywords Quantum spin systems · Phase diagrams · Mean field theory · Large deviations $\cdot$ Random current representation $\cdot$ Ising model $\cdot$ Feynman-Kac transformation

\section{Introduction}

One of the simplest classical systems exhibiting phase transition is the Curie-Weiss model. In this model, $N$ Ising spins $\underline{\sigma}=\left\{\sigma_{i}= \pm 1 ; i=1, \ldots, N\right\}$, interact via the Hamiltonian

$$
H_{N}(\underline{\sigma})=-\frac{1}{2 N} \sum_{i, j=1}^{N} \sigma_{i} \sigma_{j},
$$

(C) 2008 by L. Chayes, N. Crawford, D. Ioffe, A. Levit. Reproduction, by any means, of the entire article for non-commercial purposes is permitted without charge.

The research of Nicholas Crawford, Dmitry Ioffe and Anna Levit was partly supported by the German-Israeli Foundation under the grant I-870-58.6/2005.

L. Chayes

Department of Mathematics, University of California at Los Angeles, Los Angeles, USA

N. Crawford ( $\square)$

Department of Statistics, University of California at Berkeley, Berkeley, USA

e-mail: crawford@stat.berkeley.edu

D. Ioffe · A. Levit

Department of Industrial Engineering, The Technion, Haifa, Israel 
where the normalization by $1 / N$ makes $H_{N}$ a quantity of order $N$. (Generalizations to multiple-spin interactions may also be considered; cf. Sect. 3. However, for the time being, (1.1) will suffice.)

As is well known for the spins distributed according to the measure $\mu^{N, \beta}(\{\underline{\sigma}\}) \propto$ $\mathrm{e}^{-\beta H_{N}(\underline{\sigma})}$, i.e., in the canonical ensemble, as $N \rightarrow \infty$ the law of the empirical mean $m_{N}(\underline{\sigma})=N^{-1} \sum_{i} \sigma_{i}$ converges to a mixture of point masses at $\pm m_{\star}$ where $m_{\star}=m_{\star}(\beta)$ is the so called spontaneous magnetization. The phase transition in this model is manifestly seen from the observation that $m_{\star}(\beta) \equiv 0$ for $\beta \leq \beta_{c}$ while $m_{\star}(\beta)>0$ for $\beta>\beta_{c}$. The function $\beta \mapsto m_{\star}(\beta)$ is in fact the maximal non-negative solution of the equation

$$
m=\tanh (\beta m),
$$

while $\beta_{c}=1$ is the so called critical temperature.

The main reason why the Curie-Weiss model is so approachable is the fact that the Hamiltonian is, to within an additive constant, equal to $-\frac{1}{2} N m_{N}(\underline{\sigma})^{2}$. This permits a very explicit expression for the law of $m_{N}$ whose concentration properties are then readily controlled by straightforward large-deviation arguments. However, this simple strategy breaks down once inhomogeneous terms (i.e., those not invariant under exchanges of the spins) are added to the Hamiltonian. One example where this happens is the Curie-Weiss system in random external field where the term $\sum_{i} h_{i} \sigma_{i}$, with $h_{i}$ sampled from an i.i.d. law with zero mean, is added to $H_{N}$. While rigorous analysis is still possible in this case, the technical difficulties involved are more substantial. Significantly more complex is the Sherrington-Kirkpatrick version of (1.1), where the term $\sigma_{i} \sigma_{j}$ is weighed by a (fixed) random number $J_{x, y}$ that has been sampled from a symmetric distribution on $\mathbb{R}$. This model possesses a beautiful underlying structure $[16,19]$ which has been harnessed mathematically only very recently [13, $20,21]$.

The goal of this paper is to study another natural generalization of the Curie-Weiss model, namely to the realm of quantum mechanics. Here the classical spin variables $\sigma_{i}$ are understood as eigenvalues of $z$-component of the triplet of Pauli matrices $\left(\hat{\sigma}^{(x)}, \hat{\sigma}^{(y)}, \hat{\sigma}^{(z)}\right)$-the generators of $\mathfrak{s u}(2)$ - acting on the one-particle Hilbert space $\mathcal{X}_{1}=\operatorname{SPAN}\{|+\rangle,|-\rangle\}$. The configuration space is replaced by the product space $\mathcal{X}_{N}=\bigotimes_{i=1}^{N} \mathcal{X}_{1}$. Classical Ising configurations $\underline{\sigma} \in\{ \pm 1\}^{N}$ generate an orthonormal basis $|\underline{\sigma}\rangle=\otimes\left|\sigma_{i}\right\rangle$ of $\mathcal{X}_{N}$. If $A$ is an operator on $\mathcal{X}_{1}$ (e.g. $A=\hat{\sigma}^{(x)}$ or $A=\hat{\sigma}^{(z)}$ ) then its copy $A_{i}$ acts on $i$-th spin component of a product vector $|\underline{\varphi}\rangle=\otimes_{i=1}^{N}\left|\varphi_{i}\right\rangle \in \mathcal{X}_{N}$ via

$$
A_{i}|\underline{\varphi}\rangle=\left|\varphi_{1}\right\rangle \otimes \cdots \otimes A\left|\varphi_{i}\right\rangle \otimes \cdots \otimes\left|\varphi_{N}\right\rangle .
$$

There are at least two natural ways to introduce quantum effects into the Curie-Weiss model. Either one may make the interaction term isotropic - this corresponds to the quantum Heisenberg model-or one may consider an external transverse field. Here we focus on the latter situation: The Hamiltonian is now an operator on $\mathcal{X}_{N}$ defined by

$$
\hat{\mathcal{H}}_{N}=-\frac{1}{2 N} \sum_{i, j=1}^{N} \hat{\sigma}_{i}^{(z)} \hat{\sigma}_{j}^{(z)}-\sum_{i=1}^{N}\left(h \hat{\sigma}_{i}^{(z)}+\lambda \hat{\sigma}_{i}^{(x)}\right) .
$$

Instead of Gibbs probability measures one studies a $K M S$ state $\langle-\rangle_{\lambda, h}^{N, \beta}$ which is a positive linear functional on the $C^{\star}$-algebra of operators on $\mathcal{X}_{N}$ defined by

$$
\langle A\rangle_{\lambda, h}^{N, \beta}=\frac{\operatorname{Tr}\left(A \mathrm{e}^{-\beta \mathcal{H}_{N}}\right)}{\operatorname{Tr}\left(\mathrm{e}^{-\beta \mathcal{H}_{N}}\right)} .
$$


As before, the parameter $\beta$ plays the role of inverse temperature while $\lambda$, which corresponds to the strength of an external field, determines the overall strength of the quantum perturbation.

In particular, (1.5) gives rise to a probability distribution $\mu_{\lambda, h}^{N, \beta}$ on classical spin configurations $\underline{\sigma} \in\{ \pm 1\}^{N}$,

$$
\mu_{\lambda, h}^{N, \beta}[\underline{\sigma}]=\frac{\left\langle\underline{\sigma}\left|\mathrm{e}^{-\beta \mathcal{H}_{N}}\right| \underline{\sigma}\right\rangle}{\operatorname{Tr}\left(\mathrm{e}^{-\beta \mathcal{H}_{N}}\right)},
$$

and one can try to read a signature of phase transition in terms of concentration properties of the latter as $N$ tends to infinity.

Despite this relatively clean formulation, the quantum nature makes this model very different from the classical one. Indeed, the Hamiltonian is not simultaneously diagonalizable with the $\hat{\sigma}_{i}^{(z)}$ 's for $\lambda \neq 0$, so the very notion of "value of spin at $i$ " is apparently lost. In fact, as we explain below, a path integral approach or, equivalently, a stochastic geometric representation of $\mu_{\lambda, h}^{N, \beta}$ reveals that relevant large deviation and measure concentration analysis in the genuine quantum case $\lambda>0$ should be lifted to infinite dimensions.

We refer to $[4,5,7,17,18]$ where the ideas of stochastic geometric representation in question were originally developed, as well as to a recent review [14]. The bottom line is the representation $\mu_{\lambda, h}^{N, \beta}$ : Let $\mathbb{S}_{\beta}$ be the circle of circumference $\beta$. There exists a shift invariant probability measure $\mu_{\lambda}^{\beta}$ on piece-wise constant trajectories $\sigma: \mathbb{S}_{\beta} \mapsto\{ \pm 1\}$, such that

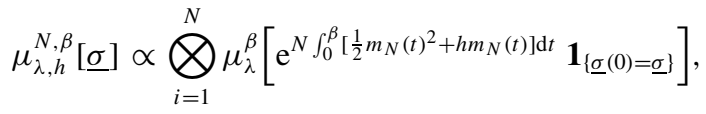

where $\underline{\sigma}(t)=\left(\sigma_{1}(t), \ldots, \sigma_{N}(t)\right)$ and $m_{N}(t)=N^{-1} \sum_{i} \sigma_{i}(t)$.

With such a representation in place, we then apply methods of large-deviation theory to derive the leading-order $N \rightarrow \infty$ asymptotic of these expectations. As can be expected, many physical properties of the quantum system may be gleaned from the properties of the minimizer of the corresponding variational problem.

The variational problem (or rather dual thereof) turns out to have intrinsic features which allow us bring methods of $F K$ percolation to bear. Many quantitative characteristics of the system will be determined explicitly (at least in the limit $N \rightarrow \infty$ ). In particular, we obtain full control of the phase diagram and the stability near optimizers for variational problem. Moreover we give an explicit characterization of the critical exponent for the decay of the optimizer as one approaches the critical curve.

Let us mention existing work on issues encompassing aspects of the present paper. The paper [10] addresses general mean field quantum spin systems in their $\mathrm{C}^{*}$ algebraic representation. Relying on an operator theoretic version of deFinetti's Theorem (i.e. Störmer's Theorem), they derive a 'mean field equation' for the extremal states of the system and formulate variational problem which these states must solve. More recently, the preprint [9] uses a slightly different path integral representation from ours to derive a qualitative probabilistic description of the solutions to these 'mean field equations'. However, neither work attempts a detailed analysis of the Quantum Curie-Weiss Model.

The remainder of this paper is organized as follows. In Sect. 2 we review the probabilistic formulation of our problem and use this opportunity to set notation. In Sect. 3 we formulate the main results of this paper. Subsequent sections are devoted to the proof of these results. 


\section{Stochastic Geometry of the Mean Field Transverse Ising Model}

Let us explicitely describe the one-circle measure $\mu_{\beta}^{\lambda}$ in (1.7). It should be noted that a natural approach, exploited in the papers Aizenman-Klein-Newman [4] and CampaninoKlein-Perez [7] among others is to realize the Gibbs state $\mu_{\beta}^{\lambda}=\langle\cdot\rangle_{\lambda, c}^{\beta}$, which we construct directly here, as the strong coupling limit of a sequence discrete Ising systems on $\mathbb{Z} / n \mathbb{Z}$. As a result of this approximation we get a weak form of ferromagnetic correlation inequalities for $\langle\cdot\rangle_{\lambda, c}^{\beta}$ for free. We will rely on this below without further mention.

We begin by introducing a convenient notation for expectation values. Given a probability measure $\mathbb{P}$ on a sample space $\Omega$ and an integrable function $f: \Omega \rightarrow \mathbb{R}$, let us denote the expectation value of $f$ by $\mathbb{P}(f)$.

Let $\mathbb{P}_{\lambda}^{\beta}$ be the distribution of the Poisson point process $\xi \subset \mathbb{S}_{\beta}$ of marks (which we visualize as punctures) on the circle $\mathbb{S}_{\beta}$ with arrival intensity $\lambda$. Given a realization of $\xi$ let us say that a classical piece-wise constant trajectory $\sigma: \mathbb{S}_{\beta} \mapsto\{ \pm 1\}$ is compatible with $\xi ; \sigma \sim \xi$, if jumps of $\sigma(\cdot)$ occur only at arrival times of $\xi_{i}$. Note that we do not require that $\sigma$ changes sign at each arrival of $\xi$. Consider now a joint probability distribution,

$$
\Phi_{\lambda, h}^{\beta}[\mathrm{d} \xi, \mathrm{d} \sigma] \propto \mathbb{P}_{\lambda}^{\beta}[\mathrm{d} \xi] \mathrm{e}^{h \int_{0}^{\beta} \sigma(t) \mathrm{d} t} \delta_{\{\sigma \sim \xi\}} .
$$

Here $\delta_{\{\sigma \sim \xi\}}$ gives mass one to $\sigma$ if it is compatible with $\xi$ and gives zero mass otherwise. In the sequel we shall suppress the sub-index $h$ whenever $h=0$. Our one-circle measure $\mu_{\lambda}^{\beta}$ is just the $\sigma$-marginal of $\Phi_{\beta}^{\lambda}$. Clearly, for $h \neq 0, \sigma$-marginals $\mu_{\lambda, h}^{\beta}$ of $\Phi_{\lambda, h}^{\beta}$ can be recovered from $\mu_{\lambda}^{\beta}$ as exponential tilts by a constant magnetic field $h$,

$$
\mu_{\lambda, h}^{\beta}[\mathrm{d} \sigma] \propto \mu_{\lambda}^{\beta}\left[\mathrm{e}^{h \int_{0}^{\beta} \sigma(t) \mathrm{d} t} \mathrm{~d} \sigma\right] .
$$

Summing with respect to compatible $\sigma$-trajectories in (2.1) we recover the $\xi$-marginal $\mathbb{Q}_{\lambda, h}^{\beta}$ of $\Phi_{\lambda}^{\beta}$ : For each realization of $\xi$ the punctured circle $\mathbb{S}_{\beta} \backslash \xi$ is a disjoint union of finite number \# $(\xi)$ of connected components,

$$
\mathbb{S}_{\beta} \backslash \xi=\bigcup_{j=1}^{\#(\xi)} I_{j}
$$

Then,

$$
\mathbb{Q}_{\lambda, h}^{\beta}[\mathrm{d} \xi] \propto \prod_{j=1}^{\#(\xi)}\left[\mathrm{e}^{h\left|I_{j}\right|}+\mathrm{e}^{-h\left|I_{j}\right|}\right] \mathbb{P}_{\lambda}^{\beta}[\mathrm{d} \xi] .
$$

Note that in the case $h=0$,

$$
\mathbb{Q}_{\lambda}^{\beta}\left[\mathrm{d} \xi_{i}\right]=\frac{2^{\#\left(\xi_{i}\right)} \mathbb{P}_{\lambda}^{\beta}\left[\mathrm{d} \xi_{i}\right]}{\mathbb{P}_{\lambda}^{\beta}\left[2^{\#\left(\xi_{i}\right)}\right]} .
$$

In general, $\left\{\mathbb{Q}_{\lambda, h}^{\beta}\right\}$ can be viewed as a family of Fortuin-Kasteleyn type random cluster measures on a graph augmented by a ghost site which represents the coupling to the external field. In particular, they possess two specific properties which we would like to stress: 
1) Stochastic domination. For each $h$ there exists an intensity $\eta=\eta(h, \lambda)$ of the Poisson process of arrivals $\xi$ on $\mathbb{S}_{\beta}$, such that

$$
\mathbb{Q}_{\lambda, h}^{\beta} \prec \mathbb{P}_{\eta}^{\beta}
$$

We refer to [2, 4] for a strategy of proving statements like (2.3), which is based on a comparison with independent Bernoulli percolation via the strong FKG inequality.

2) Edwards-Sokal representation. The spin marginal $\mu_{\lambda, h}^{\beta}$ could be recovered from $\mathbb{Q}_{\lambda, h}^{\beta}$ by the following two step procedure: First sample $\xi$ from $\mathbb{Q}_{\lambda, h}^{\beta}$, and then independently paint each connected component $I$ of $\mathbb{S}_{\beta} \backslash \xi$ into \pm 1 with probabilities $\mathrm{e}^{h|I|} /\left(\mathrm{e}^{h|I|}+\mathrm{e}^{-h|I|}\right)$ and $\mathrm{e}^{-h|I|} /\left(\mathrm{e}^{h|I|}+\mathrm{e}^{-h|I|}\right)$ respectively.

In the sequel we shall repeatedly rely on the following consequence of the two properties above: Fluctuations of $\sigma$ under $\mu_{\lambda, h}^{\beta}$ are stochastically controlled on all possible scales by arrivals of $\xi$ under the afore mentioned Poisson measure $\mathbb{P}_{\eta}^{\beta}$.

\section{Main Results}

A slight generalization of our Hamiltonian of interest is given by

$$
-\mathcal{H}_{N}=N \mathcal{P}\left(\frac{1}{N} \sum_{i=1}^{N} \sigma_{i}^{(z)}\right)+\sum_{i=1}^{N}\left[\lambda \sigma_{i}^{(x)}+h \sigma_{i}^{(z)}\right],
$$

where $\mathcal{P}(\cdot)$ is some polynomial function from $[-1,1]$ to $\mathbb{R}$.

As is apparent from (1.7), an analysis of the phase diagram of the corresponding generalized CW model in transverse field boils down to an investigation of asymptotic properties of the sequence of measures

$$
\mathbb{W}_{\lambda}^{N, \beta}(\mathrm{d} \sigma)=\frac{\otimes \mu_{\lambda}^{\beta}\left[\exp \left\{N \int_{0}^{\beta} \mathcal{P}\left(m_{N}(t)\right) \mathrm{d} t\right\} ; \mathrm{d} \sigma\right]}{\otimes \mu_{\lambda}^{\beta}\left[\exp \left\{N \int_{0}^{\beta} \mathcal{P}\left(m_{N}(t)\right) \mathrm{d} t\right\}\right]},
$$

where

$$
m_{N}(t)=\frac{1}{N} \sum_{i} \sigma_{i}(t) .
$$

This problem may be addressed with the theory of large deviations.

Theorem 3.1 For every $h \geq 0$ and $\lambda \geq 0$ the law of the average $m_{N}(\cdot)$ under the product measures $\otimes \mu_{\lambda, h}^{\beta}$ is exponentially tight on $L_{2}\left(\mathbb{S}_{\beta}\right)$, and, furthermore it satisfies on the latter space a LD principle with a good rate function $I_{\lambda, h}^{\beta}$.

This follows from fairly standard methods and will be proved in the next section. Consequently, the measures (3.2) are also exponentially tight on $L_{2}\left(\mathbb{S}_{\beta}\right)$ and they satisfy a LD principle with a good rate function $\max _{m} \mathfrak{G}_{\lambda, h}^{\beta}(m)-\mathfrak{G}_{\lambda, h}^{\beta}(\cdot)$, where,

$$
\mathfrak{G}_{\lambda, h}^{\beta}(m)=\int_{0}^{\beta} \mathcal{P}(m(t)) \mathrm{d} t-I_{\lambda, h}^{\beta}(m) .
$$


In particular, in the zero magnetic field case of $h=0$, measures (3.2) are asymptotically concentrated around solutions of

$$
\sup _{m}\left\{\int_{0}^{\beta} \mathcal{P}(m(t)) \mathrm{d} t-I_{\lambda}^{\beta}(m)\right\} \equiv \sup _{m} \mathfrak{G}_{\lambda}^{\beta}(m) .
$$

Below we shall use $(\cdot, \cdot)_{\beta}$ to denote the scalar product in $L_{2}\left(\mathbb{S}_{\beta}\right)$ and $\|\cdot\|_{\beta}$ to denote the corresponding norm. Then, since we formulate our large deviation principle in $L_{2}\left(\mathbb{S}_{\beta}\right)$,

$$
I_{\lambda, h}^{\beta}(m)=\sup _{g}\left\{(g, m)_{\beta}-\Lambda_{\lambda, h}^{\beta}(g)\right\}
$$

where

$$
\Lambda_{\lambda, h}^{\beta}(g)=\log \mu_{\lambda, h}^{\beta}\left[e^{(g, \sigma)_{\beta}}\right] .
$$

For the solution of the variational problem and, in the computation of the corresponding transitional curve, one must proceed on a case by case basis. Let us specialize to the quadratic problem.

In order to study phase coexistence in this model, we must consider the case of zero magnetic field in $z$-direction, $h=0$. Let us define

$$
\mathfrak{f}(\lambda, \beta)=\frac{1}{\beta} \operatorname{Var}_{\lambda}^{\beta}\left[(\sigma, \mathbf{1})_{\beta}\right]
$$

where $\operatorname{Var}_{\lambda}^{\beta}$ is the variance under the one-circle spin measure $\mu_{\lambda}^{\beta}$. A straightforward calculation (which we present in Sect. 6) shows that

$$
\mathfrak{f}(\lambda, \beta)=\frac{1}{\lambda} \tanh (\lambda \beta) .
$$

For the statement of our main theorem, let

$$
s_{4}^{h}(\lambda, \beta)=-\frac{\mathrm{d}^{4}}{\mathrm{~d} h^{4}} \Lambda_{\lambda}^{\beta}(h \cdot \mathbf{1}) \quad \text { and } \quad s_{4}(\lambda, \beta)=s_{4}^{0}(\lambda, \beta) .
$$

Our analysis below will show that there exists $C=C(\lambda, \beta)>0$ so that

$$
\left.\frac{\mathrm{d}^{3}}{\mathrm{~d} h^{3}}\right|_{h=h_{0}} \Lambda_{\lambda}^{\beta}(h \cdot \mathbf{1}) \leq-C h_{0}
$$

for $h_{0}$ close to zero. Thus, in view of the spin-flip symmetry and continuity of semiinvariants $s_{4}^{h}(\lambda, \beta)$ in $h, s_{4}(\lambda, \beta)$ is strictly positive.

The results of our analysis are summarized as follows:

Theorem 3.2 Let $\mathcal{P}(x)=\frac{1}{2} x^{2}$. The variational problem (3.3) at zero magnetic field $h=0$ has constant maximizers $\pm m^{*}(\lambda, \beta) \cdot \mathbf{1}$, where the spontaneous z-magnetization $m^{*}$ satisfies:

(1) If $\mathfrak{f}(\lambda, \beta) \leq 1$, then $m^{*}=0$.

(2) If $\mathfrak{f}(\lambda, \beta)>1$, then $m^{*}>0$, and, consequently there are two distinct solutions to (3.3). 
Furthermore, away from the critical curve the solutions $\pm m^{*} \cdot \mathbf{1}$ are stable in the following sense: There exists $c=c(\lambda, \beta)>0$ and a strictly convex symmetric function $U$ with $a$ $U(r) \sim r \log r$ growth at infinity, such that

$$
\mathfrak{G}_{\lambda}^{\beta}\left( \pm m^{*} \cdot \mathbf{1}\right)-\mathfrak{G}_{\lambda}^{\beta}(m) \geq c \max \left\{\left\|m \pm m^{*} \cdot \mathbf{1}\right\|_{\beta}^{2}, \int_{0}^{\beta} U\left(m^{\prime}(t)\right) \mathrm{d} t\right\},
$$

where,

$$
\left\|m \pm m^{*} \cdot \mathbf{1}\right\|_{\beta}^{2}=\min \left\{\left\|m-m^{*} \cdot \mathbf{1}\right\|_{\beta}^{2},\left\|m+m^{*} \cdot \mathbf{1}\right\|_{\beta}^{2}\right\} .
$$

As a result, the variational problem (3.3) is also stable in the supremum norm $\|\cdot\|_{\text {sup }}$. Namely, there exists a constant $c_{\text {sup }}=c_{\text {sup }}(\lambda, \beta)<\infty$, such that

$$
\mathfrak{G}_{\lambda}^{\beta}\left( \pm m^{*} \cdot \mathbf{1}\right)-\mathfrak{G}_{\lambda}^{\beta}(m) \geq \exp \left\{-\frac{c_{\text {sup }}}{\left\|m \pm m^{*} \cdot \mathbf{1}\right\|_{\text {sup }}}\right\} .
$$

Finally we have the following expression for the decay of $m^{*}>0$ in the super-critical region near the critical curve:

$$
m^{*}=m^{*}(\lambda, \beta)=\sqrt{\frac{6 \beta(\mathfrak{f}(\lambda, \beta)-1)}{s_{4}(\lambda, \beta)}}(1+o(1)),
$$

where the implicit constants depend on $\beta$ and $\lambda$ but are bounded below in compact regions of the parameter space.

Remark 1 We remark that the second term of (3.10) is particularly important in the supercritical regime $(\mathfrak{f}(\lambda, \beta)>1)$ since it rules out trajectories of $m_{N}(\cdot)$ with rapid transitions between the optimal values $\pm m^{*}$.

Remark 2 We remark that there are alternate approaches to quantum Ising systems in which the random cluster representation (and percolation therein) play a more central role, e.g. as in $[14,15]$ and [12]. While these ideas will provide a backdrop for our derivation, at the core we shall adhere to a more traditional thermodynamic/large deviations approach: Various results, e.g. stability, are stronger in this context. As a bit of foreshadowing to future work, we remark that the spin system formulation is at present essential for the extension to finite dimensional systems. On the other hand we would like to stress that our results automatically imply, as it was claimed in [15] and later on conjectured in [12], that $\mathfrak{f}(\lambda, \beta)=1$, is the equation of the critical curve for the $q=2$ quantum FK-percolation on complete graphs.

In Sect. 4 we shall prove Theorem 3.1. Then the remainder of this paper develops our proof of Theorem 3.2.

\section{Large Deviations under Product Measures}

In this section we study asymptotic properties of the average $m_{N}(\cdot)$ under the sequence of product measures $\otimes \mu_{\lambda, h}^{\beta}$. Let us begin with the proof of Theorem 3.1. 
Proof of Theorem 3.1 Let us start with the exponential tightness on $L_{2}\left(\mathbb{S}_{\beta}\right)$. Recall the following realization of compact subsets of $L_{2}\left(\mathbb{S}_{\beta}\right)$ : Let $a:[0, \beta / 2] \mapsto \mathbb{R}_{+}$be a continuous non-decreasing function with $a(0)=0$. Then,

$$
\mathcal{K}_{a}=\left\{g \in L_{2}\left(\mathbb{S}_{\beta}\right):\|g\|_{\beta} \leq 1 \text { and }\|g(s+\cdot)-g(\cdot)\|_{\beta} \leq a(s) \forall s \in[0, \beta / 2]\right\}
$$

is compact. Now, in view of the Edwards-Sokal representation,

$$
\left\|m_{N}(s+\cdot)-m_{N}(\cdot)\right\|_{\beta} \leq 2 s \frac{1}{N} \sum_{i=1}^{N} \xi_{i}\left(\mathbb{S}_{\beta}\right) .
$$

The latter expression is increasing in $\underline{\xi}=\left(\xi_{1}, \ldots, \xi_{N}\right)$, hence it is exponentially bounded in view of stochastic domination by product Poisson measures $\otimes \mathbb{P}_{\eta}^{\beta}$.

Large deviations with good rate function $I_{\lambda, h}$ defined in (3.4) is a standard consequence [6].

In the sequel we shall need the following stability estimate which implies that product measures $\otimes \mu_{\lambda, h}^{\beta}$ are sharply concentrated around constant functions.

Theorem 4.1 Let $\lambda, h \geq 0$ be fixed, and let $\eta$ be an intensity of the Poisson process of arrivals on $\mathbb{S}_{\beta}$ such that (2.3) holds. Then,

$$
I_{\lambda, h}^{\beta}(m) \geq \begin{cases}\infty, & \text { if } m \text { is not absolutely continuous } \\ \int_{0}^{\beta} U_{\eta}\left(m^{\prime}(t)\right) \mathrm{d} t, & \text { otherwise }\end{cases}
$$

where $U_{\eta}$ is an even smooth strictly convex function with a superlinear growth at infinity.

Remark 3 The function $U_{\eta}$ is defined in (4.6) below. Note that it is negative in a neighbourhood of the origin. What is important, however, is a super-linear growth at infinity which, as we shall see in the concluding Sect. 7, controls rapid oscillations for the original variational problem (3.3).

Proof Let $\mathcal{R}=\left\{0<t_{1}<t_{2}<\cdots<t_{n}=t_{0}<\beta\right\}$ be a partition of $\mathbb{S}_{\beta}$. Consider an $n$ dimensional random vector

$$
z_{N}^{\mathcal{R}}=\left[m_{N}\left(t_{1}\right)-m_{N}\left(t_{0}\right), m_{N}\left(t_{2}\right)-m_{N}\left(t_{1}\right), \ldots, m_{N}\left(t_{n}\right)-m_{N}\left(t_{n-1}\right)\right] .
$$

Evidently, $z_{N}^{\mathcal{R}}$ satisfies a LD principle with rate function $I^{\mathcal{R}}$,

$$
I^{\mathcal{R}}\left(z_{1}, \ldots, z_{n}\right)=\max _{g_{1}, \ldots, g_{n}}\left\{\sum_{i} g_{i} z_{i}-\Lambda^{\mathcal{R}}\left(g_{1}, \ldots, g_{n}\right)\right\},
$$

where

$$
\Lambda^{\mathcal{R}}\left(g_{1}, \ldots, g_{n}\right)=\log \mu_{\lambda, h}^{\beta}\left[\mathrm{e}^{\sum_{i} g_{i}\left(\sigma\left(t_{i}\right)-\sigma\left(t_{i-1}\right)\right)}\right] .
$$

By contraction ${ }^{1}$

$$
I_{\lambda, h}^{\beta}(m) \geq I^{\mathcal{R}}\left[m\left(t_{1}\right)-m\left(t_{0}\right), \ldots, m\left(t_{n}\right)-m\left(t_{n-1}\right)\right] .
$$

\footnotetext{
${ }^{1}$ While this argument is somewhat formal, it is easily made precise by using a standard mollifying procedure.
} 
It, therefore, remains to derive an appropriate lower bound on $I^{\mathcal{R}}$. Alternatively, we may seek for an upper bound on $\Lambda^{\mathcal{R}}$. Notice that in view of the Edwards-Sokal representation,

$$
\mathrm{e}^{\sum_{i} g_{i}\left(\sigma\left(t_{i}\right)-\sigma\left(t_{i-1}\right)\right)} \leq \prod_{i}\left[1+\mathbf{1}_{\left\{\xi\left(\left[t_{i-1}, t_{i}\right]\right)>0\right\}}\left(\mathrm{e}^{2 g_{i}}+\mathrm{e}^{-2 g_{i}}\right)\right] .
$$

At this stage we are delighted since the latter expression is monotone in $\xi$. Hence, by virtue of (2.3),

$$
\mu_{\lambda, h}^{\beta}\left[\mathrm{e}^{\sum_{i} g_{i}\left(\sigma\left(t_{i}\right)-\sigma\left(t_{i-1}\right)\right)}\right] \leq \prod_{i}\left[1+\left(1-\mathrm{e}^{\eta\left(t_{i-1}-t_{i}\right)}\right)\left(\mathrm{e}^{2 g_{i}}+\mathrm{e}^{-2 g_{i}}\right)\right] .
$$

Consequently,

$$
\Lambda^{\mathcal{R}}\left(g_{1}, \ldots, g_{n}\right) \leq \eta \sum_{i}\left|t_{i}-t_{i-1}\right| H\left(g_{i}\right),
$$

where $H(g)=\left(\mathrm{e}^{2 g}+\mathrm{e}^{-2 g}\right)$. By duality,

$$
I^{\mathcal{R}}\left(z_{1}, \ldots, z_{n}\right) \geq \sum_{i}\left|t_{i}-t_{i-1}\right| U_{\eta}\left[\frac{z_{i}}{\left|t_{i}-t_{i-1}\right|}\right],
$$

with,

$$
U_{\eta}(z)=\eta H^{*}\left[\frac{z}{\eta}\right]
$$

with $H^{*}$ being the Legendre transform of $H$ (and is of course explicitly computable, as was kindly pointed out to us by an anonymous referee). Thus, $U_{\eta}$ is an even smooth strictly convex function. Furthermore $U_{\eta}(m) \sim|m| \log |m|$ at infinity. Note, however, that $U_{\eta}$ is negative in a neighbourhood of the origin, in particular $U_{\eta}(0)=-2 \eta$. Going back to (4.5) we obtain that the rate function $I_{\lambda, h}^{\beta}$ satisfies

$$
I_{\lambda, h}^{\beta}(m) \geq \sum_{i}\left|t_{i}-t_{i-1}\right| U_{\eta}\left[\frac{m\left(t_{i}\right)-m\left(t_{i-1}\right)}{\left|t_{i}-t_{i-1}\right|}\right],
$$

for every partition $\mathcal{R}$ of $\mathbb{S}_{\beta}$. Taking supremum over $\mathcal{R}$-s we recover (4.2)

\section{Duality and Reduction to One-Dimensional Variational Problem}

Recall that we are restricting attention to the case of quadratic interaction $\mathcal{P}(x)=x^{2} / 2$. In particular, the functional

$$
\Psi(m)=\frac{1}{2} \int_{0}^{\beta} m^{2}(t) \mathrm{d} t=\frac{1}{2}\|m\|_{\beta}^{2}
$$

is convex and Gateaux differentiable on $L_{2}\left(\mathbb{S}_{\beta}, \mathrm{d} t\right)$. Since, as we already know, $I_{\lambda}$ has compact level sets, the supremum in (3.3) is attained. Let $\bar{m}$ be a solution,

$$
\Psi(\bar{m})-I_{\lambda}^{\beta}(\bar{m})=\max _{m}\left\{\Psi(m)-I_{\lambda}^{\beta}(m)\right\} .
$$


Then,

$$
I_{\lambda}^{\beta}(m)-I_{\lambda}^{\beta}(\bar{m}) \geq \Psi(m)-\Psi(\bar{m}) \geq(m-\bar{m}, \bar{m})_{\beta},
$$

for every $m \in L_{2}\left(\mathbb{S}_{\beta}\right)$. It follows that $I_{\lambda}^{\beta}$ is sub-differential at $\bar{m}$ and, moreover, $\bar{m} \in \partial I_{\lambda}^{\beta}(\bar{m})$. But that means $\Lambda_{\lambda}^{\beta}(\bar{m})+I_{\lambda}^{\beta}(\bar{m})=\|\bar{m}\|_{\beta}^{2}$. Hence, for every $m$ and $h$,

$$
\Lambda_{\lambda}^{\beta}(\bar{m})-\frac{1}{2}\|\bar{m}\|_{\beta}^{2} \geq \Psi(m)-I_{\lambda}^{\beta}(m)=\left\{(h, m)_{\beta}-I_{\lambda}^{\beta}(m)\right\}-\left\{(h, m)_{\beta}-\Psi(m)\right\} .
$$

We conclude: If $\bar{m}$ is a solution of (3.3), then

$$
\Lambda_{\lambda}^{\beta}(\bar{m})-\frac{1}{2}\|\bar{m}\|_{\beta}^{2}=\max _{h}\left\{\Lambda_{\lambda}^{\beta}(h)-\frac{1}{2}\|h\|_{\beta}^{2}\right\} .
$$

Since $\Lambda_{\lambda}^{\beta}$ is Gateaux differentiable and, in particular, it is everywhere sub-differentiable the reverse conclusion is equally true: if $\bar{m}$ is a solution to (5.1) then it is a solution of (3.3). Note that far reaching generalizations of the above arguments may be found in [22]. An application for general interactions $\mathcal{P}$ will be attended elsewhere. Here we continue to stick to the quadratic case. Also, since some of the computations below will be done in a greater generality, we shall casually rely on the fact that $\bar{m}$ is a solution to (3.3) if and only if it is a solution of the dual problem (5.1). The latter happens to be more susceptible to analysis.

Our next step is to assemble facts which reduce (5.1) to a study of constant fields $h=c \cdot \mathbf{1}$ for $c \in \mathbb{R}$. In what follows we rely only on the fact that the polynomial function $\mathcal{P}$ has a super-linear growth at infinity. In particular, classical Legendre transform $\mathcal{P}^{*}$ is a well defined function on $\mathbb{R}$. Let integral functional $\Psi: L_{2}\left(\mathbb{S}_{\beta}\right) \rightarrow \mathbb{R}$ be defined by

$$
\Psi(m)=\int_{0}^{\beta} \mathcal{P}\left(m_{t}\right) \mathrm{d} t,
$$

and for $h \in L^{2}\left(\mathbb{S}_{\beta}, \mathrm{d} t\right)$ let

$$
\Psi^{*}(h)=\sup _{m \in \mathcal{B}}\left\{(h, m)_{\beta}-\Psi(m)\right\}=\int_{0}^{\beta} \mathcal{P}^{*}\left(h_{t}\right) \mathrm{d} t
$$

be its Fenchel transform.

Lemma 5.1 For any polynomial $\mathcal{P}(x)$ of a super-linear growth at infinity, let $\Psi$ and $\Psi^{*}$ be defined as above. Then we have

$$
\sup _{h \in L_{2}\left(\mathbb{S}_{\beta}\right)}\left\{\Lambda_{\lambda}^{\beta}(h)-\Psi^{*}(h)\right\}=\sup _{c \in \mathbb{R}}\left\{\Lambda_{\lambda}^{\beta}(c \cdot \mathbf{1})-\beta \mathcal{P}^{*}(c)\right\} .
$$

In particular this lemma implies that the constant function $c \cdot \mathbf{1}$ optimizes the left hand side if and only if $c$ optimizes the right hand side.

To prove Lemma 5.1, we need the following observation: For any $h \in L_{2}\left(\mathbb{S}_{\beta}\right)$, let $h_{l}$ and $h_{r}$ be the pair functions obtained by reflecting $h$ about 0 and $\frac{\beta}{2}$. That is let

$$
h_{l}(t)= \begin{cases}h(t) & \text { if } t \in\left[0, \frac{\beta}{2}\right] \\ h(\beta-t) & \text { if } t \in\left[\frac{\beta}{2}, \beta\right]\end{cases}
$$

and $h_{r}$ be defined analogously. 
Lemma 5.2 Let $h \in L_{2}\left(\mathbb{S}_{\beta}\right)$ and $h_{l}, h_{r}$ be as above. Then we have

$$
\Lambda_{\lambda}^{\beta}(h) \leq \frac{1}{2}\left[\Lambda_{\lambda}^{\beta}\left(h_{l}\right)+\Lambda_{\lambda}^{\beta}\left(h_{r}\right)\right] .
$$

Consequently, for every $h \in L_{2}\left(\mathbb{S}_{\beta}\right)$,

$$
\Lambda_{\lambda}^{\beta}(h) \leq \frac{1}{\beta} \int_{0}^{\beta} \Lambda_{\lambda}^{\beta}(h(t) \cdot \mathbf{1}) \mathrm{d} t .
$$

We note that Lemma 5.2 was originally proved in a somewhat more general (but completely analogous) context in [9].

Proof Inequality (5.6) follows from reflection positivity of classical ferromagnetic Ising systems: Recall that $\mu_{\lambda}^{\beta}$ is the continuum limit of a sequence of discrete Ising models with asymptotically singular interactions. Let $M \in \mathbb{N}$ be fixed and suppose that the field $h \in \mathcal{D}$, that is $h$ is piecewise constant on the dyadic intervals $\left[\frac{k}{2^{M}} \beta, \frac{k+1}{2^{M}} \beta\right.$ ). Expressing the discrete Ising model on a circle via transfer matrices and using the generalized Cauchy-Schwartz inequality [11], one obtains (5.6) directly whenever the field $h \in \mathcal{D}$. Passing to limits with respect to the discrete Ising models, we immediately have (5.6) for any dyadic piecewise constant function $h$. Standard approximation arguments extend the bound to the general case. Finally, (5.7) follows from repeated applications of (5.6)

Let us go back to the proof of Lemma 5.1. By (5.3) and (5.7),

$$
\Lambda_{\lambda}^{\beta}(h)-\Psi^{*}(h) \leq \int_{0}^{\beta}\left(\frac{1}{\beta} \Lambda_{\lambda}^{\beta}(h(t) \cdot \mathbf{1})-\mathcal{P}^{*}(h(t))\right) \mathrm{d} t
$$

(5.4) follows.

\section{Analysis and Stability of the One-Dimensional Problem}

Following (5.4) we need to analyze the following one-dimensional problem,

$$
\sup _{c} \mathfrak{g}_{\lambda}^{\beta}(c) \equiv \sup _{c \in \mathbb{R}}\left\{\Lambda_{\lambda}^{\beta}(c \cdot \mathbf{1})-\frac{\beta c^{2}}{2}\right\} .
$$

Evidently, $\left|\Lambda_{\lambda}^{\beta}(c \cdot \mathbf{1})\right| \leq \beta|c|$. Hence,

$$
\lim _{|c| \rightarrow \infty} \Lambda_{\lambda}^{\beta}(c \cdot \mathbf{1})-\frac{\beta c^{2}}{2}=-\infty
$$

so that solutions to (6.1) always exist. Any such solution $c$ is a critical point satisfying

$$
c=\frac{1}{\beta} \frac{\mathrm{d}}{\mathrm{d} c} \Lambda_{\lambda}^{\beta}(c \cdot \mathbf{1})=\frac{1}{\beta} \mu_{\lambda, c}^{\beta}\left[(\sigma, \mathbf{1})_{\beta}\right] \equiv M(c) .
$$

By the FKG and GHS inequalities (cf. the discussion at the beginning of Sect. 2) the function $\frac{\mathrm{d}}{\mathrm{d} c} M(c)=\frac{1}{\beta} \operatorname{Var}_{\lambda, c}^{\beta}\left((\sigma, \mathbf{1})_{\beta}\right)$ is positive and decreasing in $c$. In fact, as we see below, it is 
strictly decreasing on $[0, \infty)$. Therefore (6.3) has nontrivial solutions if and only if

$$
\left.\frac{\mathrm{d}}{\mathrm{d} c} M(c)\right|_{c=0}=\frac{1}{\beta} \operatorname{Var}_{\lambda}^{\beta}\left[(\sigma, \mathbf{1})_{\beta}\right]>1 .
$$

As $\left\langle(\sigma, \mathbf{1})_{\beta}\right\rangle_{\lambda}^{\beta}=0$ by spin flip symmetry, $\operatorname{Var}_{\lambda}^{\beta}\left[(\sigma, \mathbf{1})_{\beta}\right]=\mu_{\lambda}^{\beta}\left((\sigma, \mathbf{1})_{\beta}^{2}\right)$.

We are reduced to computing

$$
\mu_{\lambda}^{\beta}\left((\sigma, \mathbf{1})_{\beta}^{2}\right)=\beta \int_{0}^{\beta} \mu_{\lambda}^{\beta}\left(\sigma_{0} \sigma_{t}\right) \mathrm{d} t,
$$

where we have used rotational invariance in the equality. In view of the Edwards-Sokal representation, the \pm 1 symmetry implies

$$
\mu_{\lambda}^{\beta}\left(\sigma_{0} \sigma_{t}\right)=\frac{\mathbb{P}_{\lambda}^{\beta}\left[0 \leftrightarrow t ; 2^{\#(\xi)}\right]}{\mathbb{P}_{\lambda}^{\beta}\left[2^{\#(\xi)}\right]},
$$

where, as in (2.2) before, \#( $\xi$ ) denotes the number of connected components determined by the underlying Poisson process and $\{0 \leftrightarrow t\}$ denotes the event the 0 and $t$ are in the same connected component of the complement of the arrival points.

There are two cases to consider: either there is an arrival in $[t, \beta]$ or there is not. Taking this under consideration, we have

$$
\mathbb{P}_{\lambda}^{\beta}\left[0 \leftrightarrow t ; 2^{\#(\xi)}\right]=e^{\lambda(\beta-2 t)}+e^{-\lambda(\beta-2 t)} .
$$

A simple computation shows

$$
\mathbb{P}_{\lambda}^{\beta}\left[2^{\#(\xi)}\right]=e^{-\lambda \beta}+e^{\lambda \beta} .
$$

Upon integrating, we find

$$
\frac{1}{\beta} \operatorname{Var}_{\lambda}^{\beta}\left((\sigma, \mathbf{1})_{\beta}\right)=\frac{1}{\lambda} \tanh (\lambda \beta)
$$

Conclusions (1) and (2) of Theorem 3.2 follow as soon as we show that

$$
\mathfrak{v}_{\lambda}^{\beta}(c) \equiv \frac{1}{\beta} \operatorname{Var}_{\lambda, c}^{\beta}\left((\sigma, \mathbf{1})_{\beta}\right)
$$

is indeed strictly decreasing on $[0, \infty)$. To this end, note that,

$$
\frac{\mathrm{d} \mathfrak{v}_{\lambda}^{\beta}(c)}{\mathrm{d} c}=\int_{0}^{\beta} \int_{0}^{\beta} \int_{0}^{\beta} U_{\lambda, c}^{\beta}(r, s, t) \mathrm{d} r \mathrm{~d} s \mathrm{~d} t,
$$

where,

$$
\begin{aligned}
U_{\lambda, c}^{\beta}(r, s, t)= & \left\langle\left.\sigma(r) \sigma(s) \sigma(t)\right|_{\lambda, c} ^{\beta}-\left\langle\sigma ( r ) \sigma ( s ) | _ { \lambda , c } ^ { \beta } \left\langle\left.\sigma(t)\right|_{\lambda, c} ^{\beta}-\left\langle\sigma ( s ) \sigma ( t ) | _ { \lambda , c } ^ { \beta } \left\langle\left.\sigma(r)\right|_{\lambda, c} ^{\beta}\right.\right.\right.\right.\right. \\
& -\left\langle\sigma ( r ) \sigma ( t ) | _ { \lambda , c } ^ { \beta } \left\langle\left.\sigma(s)\right|_{\lambda, c} ^{\beta}+2\left\langle\sigma ( r ) | _ { \lambda , c } ^ { \beta } \left\langle\sigma ( s ) | _ { \lambda , c } ^ { \beta } \left\langle\left.\sigma(t)\right|_{\lambda, c} ^{\beta}\right.\right.\right.\right.\right.
\end{aligned}
$$

the third Ursell function for $\mu_{\lambda, c}^{\beta}$. In view of the above mentioned identification of $\mu_{\lambda, c}^{\beta}$ as a limit of one-dimensional ferromagnetic Ising models, we state the following result without 
proof: For any $c \geq 0$,

$$
U_{\lambda, c}^{\beta}(r, s, t) \leq 0 \quad \text { for all } r, s, t \in \mathbb{S}_{\beta} .
$$

In order to derive a strict bound we shall employ a similar approximation, which will allow us to transfer the random current representation used by Aizenman [1] and AizenmanFernandez [3] to this continuous time setting. We obtain the following lemma, whose proof is relegated to the Appendix:

Lemma 6.1 (Random Current Bound) For every $\lambda \geq 0$ and $\beta<\infty$ there exists $C_{1}=$ $C_{1}(\lambda, \beta)>0$ such that for any positive constant field $c$ and any triplet of points $r, s, t \in \mathbb{S}_{\beta}$, we have the following bound:

$$
U_{\lambda, c}^{\beta}(r, s, t) \leq-C_{1}(\lambda, \beta) \mathrm{e}^{-2 c \beta} .
$$

Thereby, the phase diagram (6.4) is justified.

Let us turn to the stability issue: As follows from (6.10) and (6.13), there exists $\chi=$ $\chi(\lambda, \beta)$, such that for any $c \geq 0$,

$$
\frac{\mathrm{d} \mathfrak{v}_{\lambda}^{\beta}(c)}{\mathrm{d} c} \leq-\chi\left\langle\sigma_{0}\right\rangle_{\lambda, c}^{\beta}
$$

Remark 4 Actually, the bound (6.14) could have been obtained by direct computation (with $2 \times 2$ matrices). Nevertheless, we found it instructive to derive (6.14) using stochastic geometric methods behind Lemma 6.1, as these tools may be brought to bare in more general systems (e.g. see [8] for a formulation of the 'Switching Lemma' for quantum Ising systems.)

This way or another, inequality (6.14) implies: For any $0 \leq c_{1}<c_{2}$,

$$
\chi\left\langle\sigma_{0}\right\rangle_{\lambda, c_{1}}^{\beta}\left(c_{2}-c_{1}\right) \leq \mathfrak{v}_{\lambda}^{\beta}\left(c_{1}\right)-\mathfrak{v}_{\lambda}^{\beta}\left(c_{2}\right) \leq \chi\left\langle\sigma_{0}\right\rangle_{\lambda, c_{2}}^{\beta}\left(c_{2}-c_{1}\right) .
$$

Assume that (6.4) holds, and let $c^{*}=m^{*}>0$ be the positive maximizer of $\mathfrak{g}_{\lambda}^{\beta}$. Then, of course, $0=\frac{\mathrm{d}}{\mathrm{d} c} \mathfrak{g}_{\lambda}^{\beta}\left(c^{*}\right)$. Let us derive an upper bound on

$$
\frac{\mathrm{d}}{\mathrm{d} c} \mathfrak{g}_{\lambda}^{\beta}(c)=\beta c\left[\frac{1}{c} \int_{0}^{c} \mathfrak{v}_{\lambda}^{\beta}(\tau) \mathrm{d} \tau-1\right]
$$

For $c>c^{*}$,

$$
\frac{1}{c} \int_{0}^{c} \mathfrak{v}_{\lambda}^{\beta}(\tau) \mathrm{d} \tau=\frac{1}{c^{*}} \int_{0}^{c^{*}} \mathfrak{v}_{\lambda}^{\beta}\left[\tau \frac{c}{c^{*}}\right] \mathrm{d} \tau=1+\frac{1}{c^{*}} \int_{0}^{c^{*}}\left\{\mathfrak{v}_{\lambda}^{\beta}\left[\tau \frac{c}{c^{*}}\right]-\mathfrak{v}_{\lambda}^{\beta}(\tau)\right\} \mathrm{d} \tau .
$$

By (6.15) the second term is bounded above by

$$
\begin{aligned}
& -\chi \int_{0}^{c^{*}}\left\langle\sigma_{0}\right\rangle_{\lambda, \tau}^{\beta}\left[\frac{c}{c^{*}}-1\right] \tau \mathrm{d} \tau \\
& =-\chi\left(c-c^{*}\right) \frac{1}{c^{*}} \int_{0}^{c^{*}}\left\langle\sigma_{0}\right\rangle_{\lambda, \tau}^{\beta} \tau \mathrm{d} \tau \leq-\frac{\chi\left(c^{*}\right)^{2}}{3}\left(c-c^{*}\right),
\end{aligned}
$$

where at the last step we have relied on $\left\langle\sigma_{0}\right\rangle_{\lambda, \tau} \geq \tau$ for every $\tau \leq c^{*}$. 
In a completely similar fashion we deduce that,

$$
\frac{1}{c} \int_{0}^{c} \mathfrak{v}_{\lambda}^{\beta}(\tau) \mathrm{d} \tau-1 \geq \frac{\chi\left(c^{*}\right)^{2}}{3}\left(c^{*}-c\right),
$$

whenever, $c \leq c^{*}$. We have proved:

Lemma 6.2 Assume (6.4). The positive maximizer $m^{*}=c^{*}>0$ of $\mathfrak{g}_{\lambda}^{\beta}$ is stable in the following sense: For every $c \in[0, \infty)$,

$$
\mathfrak{g}_{\lambda}^{\beta}\left(c^{*}\right)-\mathfrak{g}_{\lambda}^{\beta}(c) \geq \frac{\chi \beta\left(c^{*}\right)^{3}}{24}\left(c-c^{*}\right)^{2} \equiv d\left(c-c^{*}\right) .
$$

\section{Conclusion of the Proof of Theorem 3.2: Claims (3.10) and (3.12)}

Let us return first to the infinite dimensional variational problem (5.1). The FKG property of $\mu_{\lambda}^{\beta}$ (inherited in a limit of ferromagnetic Ising models) evidently implies that $\Lambda_{\lambda}^{\beta}(h) \leq$ $\Lambda_{\lambda}^{\beta}(|h|)$. Since by (5.8),

$$
\Lambda_{\lambda}^{\beta}(|h|)-\frac{1}{2}\|h\|_{\beta}^{2} \leq \frac{1}{\beta} \int_{0}^{\beta} \mathfrak{g}_{\lambda}^{\beta}(|h(t)|) \mathrm{d} t,
$$

we readily recover from (6.18) the following $L_{2}$-stability bound:

$$
\begin{gathered}
\left\{\Lambda_{\lambda}^{\beta}\left(m^{*} \cdot \mathbf{1}\right)-\frac{\beta\left(m^{*}\right)^{2}}{2}\right\}-\left\{\Lambda_{\lambda}^{\beta}(|h|)-\frac{1}{2}\|h\|_{2 ; \beta}^{2}\right\} \\
\geq \frac{1}{\beta} \int_{0}^{\beta} d\left(|h(t)|-m^{*}\right) \mathrm{d} t \equiv D(h) .
\end{gathered}
$$

In order to transfer this bound to the original variational problem (3.3) we shall again rely on duality considerations of Sect. 5. Recall that

$$
\Lambda_{\lambda}^{\beta}\left(m^{*} \cdot \mathbf{1}\right)-\beta\left(m^{*}\right)^{2} / 2=\beta\left(m^{*}\right)^{2} / 2-I_{\lambda}^{\beta}\left(m^{*} \cdot \mathbf{1}\right) .
$$

Therefore, by (7.1)

$$
\beta\left(m^{*}\right)^{2} / 2-I_{\lambda}^{\beta}\left(m^{*} \cdot \mathbf{1}\right) \geq\left\{(h, m)_{\beta}-\|h\|_{\beta}^{2} / 2\right\}-I_{\lambda}^{\beta}(m)+D(h) .
$$

Taking $h=m$ we arrive at

$$
\mathfrak{G}_{\lambda}^{\beta}\left(m^{*} \cdot \mathbf{1}\right)-\mathfrak{G}_{\lambda}^{\beta}(m) \geq D(m) .
$$

Obviously, there exists $c_{1}=c_{1}(\lambda, \beta)$, such that

$$
D(m) \geq c_{1}\left\|m \pm m^{*} \cdot \mathbf{1}\right\|_{\beta}^{2} .
$$

Thereby, the first term in (3.10) is recovered. In order to recover the second term in (3.10), set $\bar{m}=m^{*} \cdot \mathbf{1}$ and just compute:

$$
\mathfrak{G}_{\lambda}^{\beta}(\bar{m})-\mathfrak{G}_{\lambda}^{\beta}(m)=\left\{\frac{1}{2}\|\bar{m}\|_{\beta}^{2}-\frac{1}{2}\|m\|_{\beta}^{2}\right\}-\left\{I_{\lambda}^{\beta}(\bar{m})-I_{\lambda}^{\beta}(m)\right\}
$$




$$
\begin{aligned}
= & \frac{1}{2}\|\bar{m}\|_{\beta}^{2}-\frac{1}{2}\|m\|_{\beta}^{2}+(\bar{m}, m-\bar{m})_{\beta} \\
& +I_{\lambda}^{\beta}(m)-I_{\lambda}^{\beta}(\bar{m})-(\bar{m}, m-\bar{m})_{\beta} \\
= & -\frac{1}{2}\|m-\bar{m}\|_{\beta}^{2}+I_{\lambda, m^{*}}^{\beta}(m) .
\end{aligned}
$$

Repeating the same computation with $-\bar{m}$, we infer:

$$
\begin{aligned}
\mathfrak{G}_{\lambda}^{\beta}(\bar{m})-\mathfrak{G}_{\lambda}^{\beta}(m) & \geq-\frac{1}{2}\|m \pm \bar{m}\|_{\beta}^{2}+\min \left\{I_{\lambda, m^{*}}^{\beta}(m), I_{\lambda,-m^{*}}^{\beta}(m)\right\} \\
& \geq-\frac{1}{2}\|m \pm \bar{m}\|_{\beta}^{2}+\int_{0}^{\beta} U_{\eta\left(\lambda, m^{*}\right)}\left(m^{\prime}(t)\right) \mathrm{d} t,
\end{aligned}
$$

as follows from (4.2). At this point we recall (7.4), and the full statement of (3.10) becomes an easy exercise (with $U=U_{\eta}$ ).

Let now $m$ be such that $\|m \pm \bar{m}\|_{\text {sup }}=2 \delta$. Let us consider only the case when $\max _{t} m(t)=m^{*}+2 \delta$. If $\min _{t} m(t)>\delta$, then $\|m \pm \bar{m}\|_{\beta}^{2} \geq \delta^{2} \beta$. Otherwise, without loss of generality, we may assume that $m(0)=m^{*}+2 \delta$ and let $r=\min \left\{t>0: m(t)=m^{*}+\delta\right\}$. Obviously, in such circumstances,

$$
\|m \pm \bar{m}\|_{\beta}^{2} \geq r \delta^{2} \quad \text { whereas } \quad \int_{0}^{\beta} U\left(m^{\prime}(t)\right) \mathrm{d} t \geq \int_{0}^{r} U\left(m^{\prime}(t)\right) \mathrm{d} t-2 \eta \beta .
$$

However, by convexity,

$$
\int_{0}^{r} U\left(m^{\prime}(t)\right) \mathrm{d} t \geq r U(\delta / r) \sim \delta \log (1 / r)
$$

for $r \ll \delta$. Therefore, the $\int_{0}^{\beta} U\left(m^{\prime}(t)\right) \mathrm{d} t$-term in $\max \left\{\|m \pm \bar{m}\|_{\beta}^{2}, \int_{0}^{\beta} U\left(m^{\prime}(t)\right) \mathrm{d} t\right\}$ becomes dominant for $r \sim \mathrm{e}^{-1 / \delta}$. Hence (3.11).

Finally, consider the claim (3.12). As we have seen in Sect. 5, $m^{*} \cdot 1$ solves the dual variational problem (5.1). In particular,

$$
m^{*}=\frac{1}{\beta} \mu_{\lambda, m^{*}}^{\beta}\left((\sigma, \mathbf{1})_{\beta}\right) .
$$

Via the Taylor expansion we may write

$$
m^{*}=\frac{m^{*}}{\beta} \operatorname{Var}_{\lambda, 0}^{\beta}\left((\sigma, \mathbf{1})_{\beta}\right)-\frac{s_{4}(\lambda, \beta)\left(m^{*}\right)^{3}}{6 \beta}+o\left(\left(m^{*}\right)^{4}\right)
$$

noting that odd derivatives of $\Lambda_{\lambda}^{\beta}$ vanish at zero by spin flip symmetry. As we have already mentioned, Lemma 6.1 implies that $s_{4}(\lambda, \beta)>0$. Claim (3.12) follows easily.

\section{Appendix}

Proof of Lemma 6.1 We begin by recalling the random current representation of the third Ursell function for a discrete Ising model, see [3]. 
Following the notation of [3], consider a general finite graph Ising model on a vertex set $V$ (also called sites), elements of which we denote with $i$, and with bonds $b$ in some fixed subset $\mathcal{E} \subset V \times V$. Let us denote the coupling constants by $J_{b}$ and local fields by $h_{i}$. It is useful to interpret the local fields $h_{i}$ as the coupling constants between the site $i$ and a 'ghost' site $\mathfrak{g}$. We augment the graph $V, \mathcal{E}$ by including $\mathfrak{g}$ along with an edge set connecting $\mathfrak{g}$ to each vertex in $V$. Let us denote the augmented edge set by $\mathcal{E}^{\prime}$.

Let $\underline{n}=\left(n_{b}\right)_{b \in \mathcal{E}^{\prime}}$ denote a sequence of integer valued 'fluxes' attached to the bonds of the underlying graph. We say that a site $i \in V$ is a source if $\sum_{i \in b} n_{b}$ is odd and denote the collection of sources other than the ghost site by $\partial \underline{n}$.

We say that $x \leftrightarrow y$ if there exists a path of non-zero fluxes connecting $x$ to $y$ using bonds in $\mathcal{E}^{\prime}$. Moreover, $x \nrightarrow \mathfrak{g}$ means that whenever $x \leftrightarrow y, n_{y, \mathfrak{g}}=0$. For any subset of bonds $\mathcal{B} \subset \mathcal{E}$, let $\langle\cdot\rangle_{\mathcal{B}}$ denote the Gibbs state for the Ising model with the constants $\left(J_{b}\right)_{b \in \mathcal{B}}$ set to 0 . Further, let

$$
W(\underline{n})=\prod_{b} \frac{J_{b}{ }^{n_{b}}}{n_{b} !}
$$

and

$$
Z=\sum_{\partial \underline{n}=\varnothing} W(\underline{n})
$$

Suppose $r, s, t \in V$ are fixed sites in the Ising model under consideration. To avoid excessive provisos hereafter we shall assume that all three points are distinct. For a collection of fluxes $\underline{n}$, let us say that $b \in C_{\underline{n}}(r)$ if $r \leftrightarrow i$ for some $i \in b$. Let $C_{\underline{n}}^{c}(r)$ be the sub-graph of $\left(V \cup \mathfrak{g}, \mathcal{E}^{\prime}\right)$ obtained by deleting all the bonds from $C_{\underline{n}}(r)$.

Then we have (the reader should consult Corollary 3.7 in [3] for a derivation)

$$
\begin{aligned}
U_{\lambda, c}^{\beta}(r, s, t)= & \left\{\sum_{\partial \underline{n}_{1}=\{r, s\}, \partial \underline{n}_{2}=\varnothing} \frac{W\left(\underline{n}_{1}\right)}{Z} \frac{W\left(\underline{n}_{2}\right)}{Z} \mathbf{1}_{\underline{n}_{1}+\underline{n}_{2}: r \nrightarrow \mathfrak{g}} \times\left[\left\langle\sigma_{t}\right\rangle_{{\underline{n}_{1}+\underline{n}_{2}}_{2}^{c}(r)}-\left\langle\sigma_{t}\right\rangle\right]\right\} \\
& +\{s \Leftrightarrow t\},
\end{aligned}
$$

where $\{s \Leftrightarrow t\}$ represents the first term with the roles of $s$ and $t$ interchanged. Here $\underline{n}_{1}$ and $\underline{n}_{2}$ are independent copies of fluxes.

Clearly, the weights $W(\underline{n})$ are proportional to the probability that a family of independent Poisson random variables indexed by (generalized) bonds take a collection of values determined by $\underline{n}$. We need to differentiate between the random variables associated to the bonds of the graph and the bonds with the ghost site $\mathfrak{g}$. Specifically, let $\left\{\mathcal{N}_{b}, \mathcal{M}_{i}\right\}_{b \in \mathcal{E}, i \in V}$ denote a collection of independent Poisson variables with respective parameters $\left\{J_{b}, h_{i}\right\}_{b \in \mathcal{E}, i \in V}$ and let $\mathbb{P}$ denote the joint probability measure associated to these processes.

It is well known that if $h \geq 0$, spin correlations are increasing with respect to coupling strengths, so each summand on the right-hand side of (8.3) must be non-positive. Therefore, neglecting summands we obtain

$$
\begin{aligned}
& U_{\lambda, c}^{\beta}(r, s, t) \\
& \leq \sum_{\partial \underline{n}_{1}=\{r, s\}, \partial \underline{n}_{2}=\varnothing} \frac{W\left(\underline{n}_{1}\right)}{Z} \frac{W\left(\underline{n}_{2}\right)}{Z} \mathbf{1}_{\left\{\underline{n}_{1}+\underline{n}_{2}: r \nrightarrow \mathfrak{g}\right\}} 1_{\left.\underline{\underline{n}}_{1}+\underline{n}_{2}: r \leftrightarrow t\right\}} \times\left[\left\langle\sigma_{t}\right\rangle_{C_{\underline{n}_{1}+\underline{n}_{2}}^{c}(r)}-\left\langle\sigma_{t}\right\rangle\right] \\
& \quad+\{s \Leftrightarrow t\},
\end{aligned}
$$

where we drop analogous terms in the expression $\{s \Leftrightarrow t\}$. 
Let us concentrate on the first term on the right-hand side of the inequality as the second may be treated by symmetric considerations. Note that on the set $\left\{\underline{n}_{1}+\underline{n}_{2}: r \leftrightarrow t\right\}$ the point $t$ is isolated, and hence we have

$$
\left\langle\sigma_{t}\right\rangle_{C_{\underline{n}_{1}+\underline{n}_{2}}^{c}(r)}=0
$$

by spin flip symmetry. Set

$$
E_{r, s, t}=\left\{\partial \underline{n}_{1}=\{r, s\}\right\} \cap\left\{\partial \underline{n}_{2}=\emptyset\right\} \cap\left\{\underline{n}_{1}+\underline{n}_{2}: r \nrightarrow \mathfrak{g}\right\} \cap\left\{\underline{n}_{1}+\underline{n}_{2}: r \leftrightarrow t\right\} .
$$

After the reduction made above (8.4) may be expressed as

$$
U_{\lambda, c}^{\beta}(r, s, t) \leq-\left\langle\sigma_{t}\right\rangle \frac{\mathbb{P} \otimes \mathbb{P}\left(E_{r, s, t}\right)}{\left(\mathbb{P}\left(\partial \underline{n}_{1}=\varnothing\right)\right)^{2}}+\{s \Leftrightarrow t\} .
$$

Let us now specialize to Ising models on the circle with $2^{N}$ vertices. We take coupling and field strengths given by

$$
\begin{aligned}
\mathrm{e}^{-2 J_{b}} & =\frac{\lambda \beta}{2^{N}}, \\
h_{i} & =\frac{h \beta}{2^{N}} .
\end{aligned}
$$

Without loss of generality we may assume the orientation

$$
0<r<s<t<\beta \text {. }
$$

Continuity arguments imply that it is sufficient for us prove the bound (6.13) assuming each point is of the form $\beta j / 2^{k}$ for some $j, k \in \mathbb{N}$ fixed.

It turns out to be useful to normalize the probabilities associated with the variables $\left\{\mathcal{N}_{b, 1}, \mathcal{N}_{b, 2}\right\}_{b}$ so that the event $\left\{N_{b, i}=\right.$ even $\}$ has weight 1 . Let the symbol $e^{*} e^{*}$ denote the event that the pair of Poisson variables $n_{1, b}, n_{2, b}$ are even and one of the two is non-zero. The outcomes of interest can be characterized by an ordered pair $00, e^{*} e^{*}, e o, o e, o o$. Then, at the discrete level

$$
\begin{aligned}
& \mathbb{W}(e o) \sim 1-2 \lambda \Delta, \\
& \mathbb{W}(o o) \sim 1-4 \lambda \Delta, \\
& \mathbb{W}\left(e^{*} e^{*}\right) \sim 1-4 \lambda \Delta, \\
& \mathbb{W}(00) \sim 4 \lambda \Delta,
\end{aligned}
$$

where $\Delta$ is the time step $\frac{1}{2^{N}}$. We may normalize these weights by $e^{-4 \lambda \Delta}$ so that the 'arrivals' of weights corresponding to the event 00 becomes a Poisson process of rate $4 \lambda$ in the limit as $\Delta \rightarrow 0$. We also have two independent copies of $\mathcal{M}_{i}$ to consider. For these, we leave the corresponding probabilities normalized.

We pass to the limit as $N \rightarrow \infty$ for

$$
\frac{\mathbb{P} \otimes \mathbb{P}\left(E_{r, s, t}\right)}{\left(\mathbb{P}\left(\partial \underline{n}_{1}=\varnothing\right)\right)^{2}}
$$

using the weights (8.8)-(8.11) in place of the corresponding probabilities to obtain a stochastic integration described as follows: 
Let $\left\{\zeta_{\mathfrak{g}}\right\}$ denote a Poisson process with rate $2 h$, which superposes the pair of Poisson processes associated to arrivals of bonds with $\mathfrak{g}$ into a single process. In addition, let $\phi$ denote a Poisson process of holes with rate $4 \lambda$, associated to $\mathbb{S}_{\beta}$. The entire collection of Poisson processes is taken to be jointly independent.

This process of bonds and holes partition the disjoint pair $\mathbb{S}_{\beta}$ into a collection of intervals. Each interval comes labeled with one of the four symbols $e^{*} e^{*}, e o, o e, o o$. Also, each label comes with a weight; according to (8.8)-(8.10), if the length of the interval is denote by $|I|$ then

$$
\begin{aligned}
& \mathbb{W}_{I}(\text { eo })=\mathbb{W}_{I}(\text { oe })=e^{-2 \lambda|I|-4 \lambda \beta}, \\
& \mathbb{W}_{I}\left(e^{*} e^{*}\right)=\mathbb{W}_{I}(\text { oo })=e^{-4 \lambda|I|-4 \lambda \beta} .
\end{aligned}
$$

Moreover these labels must satisfy a consistency condition defined as follows. With $v$ a configuration of labels on $\mathbb{S}_{\beta}$, let $v \sim\left(\varsigma_{\mathfrak{g}}, \phi\right)$ the condition that the labels of intervals adjacent to arrivals of $\varsigma_{\mathfrak{g}}$ consist of one taken from $\left\{e^{*} e^{*}, o o\right\}$ and one taken from $\{e o, o e\}$. Further, both intervals adjacent to an arrival of $\phi$ are labeled with $e^{*} e^{*}$. We also introduce a modified consistency $v \stackrel{r, s}{\sim}\left(\varsigma_{\mathfrak{g}}, \phi\right)$ containing the additional requirement that $v$ is discontinuous at $\{r, s\}$ with the labels of intervals adjacent to these points given by the pair $\left\{e^{*} e^{*}, o e\right\}$ or the pair $\{o o, e o\}$. Finally, let $\mathbb{W}(v)$ denote the product of the weights associated to the labels as defined in the previous paragraph.

With these preparations, we evidently have

$$
\lim _{N \rightarrow \infty} \frac{\mathbb{P}_{N} \otimes \mathbb{P}_{N}\left(E_{r, s, t}\right)}{\left(\mathbb{P}_{N}\left(\underline{n}_{1}=\varnothing\right)\right)^{2}} \geq \frac{\mathbb{E}\left[\mathbb{1}_{\left\{\zeta_{\mathfrak{g}}(C(r))=0\right\} \cap\{\phi([r, t])=0\}} \sum_{\nu \sim \sim_{\nu, s}^{r, s}\left(\zeta_{\mathfrak{g}}, \phi\right)} \mathbb{W}(\nu)\right]}{\mathbb{E}\left[\sum_{\nu \sim(\zeta \mathfrak{g}, \phi)} \mathbb{W}(\nu)\right]},
$$

where $C(r)$ denotes the connected component of $r$ in $\mathbb{S}_{\beta} \backslash \phi\left(\mathbb{S}_{\beta}\right)$.

Since for any label $\nu, e^{-4 \lambda \beta} \leq e^{4 \lambda \beta} \mathbb{W}(\nu) \leq e^{-2 \lambda \beta}$, one can bound below the right hand side in (8.15) as,

$$
e^{-2 \lambda \beta} \frac{\mathbb{E}\left[\mathbb{1}_{\left\{\zeta_{\mathfrak{g}}(C(r))=0\right\} \cap\{\phi([r, t])=0\}} \sum_{\nu \sim{ }_{\nu}, s}{ }_{\left(\zeta_{\mathfrak{g}}, \phi\right)} \mathbb{1}\right]}{\mathbb{E}\left[\sum_{\nu \sim\left(\zeta_{\mathfrak{g}}, \phi\right)} \mathbb{1}\right]} .
$$

In each integrand the sum can consist of at most two terms, so in particular, the latter expression is further bounded below by

$$
\frac{e^{-2 \lambda \beta}}{2} \mathbb{E}\left[\mathbb{1}_{\{\zeta \mathfrak{g}(C(r))=0\} \cap\{\phi([r, t])=0\}} \sum_{\substack{r, s \\ \nu \sim(\zeta \mathfrak{g}, \phi)}} \mathbb{1}\right] .
$$

It remains to notice that if $\varsigma_{\mathfrak{g}}\left(\mathbb{S}_{\beta}\right)=0$ and $\phi\left(\mathbb{S}_{\beta}\right)=0$ then there are exactly two compatible labels $v \stackrel{r, s}{\sim}\left(\varsigma_{\mathfrak{g}}, \phi\right)$. Consequently,

$$
\mathbb{E}\left[\mathbb{1}_{\left\{\zeta_{\mathfrak{g}}(C(r))=0\right\} \cap\{\phi([r, t])=0\}} \sum_{\substack{r, s \\ \nu \sim \sim(\zeta \mathfrak{g}, \phi)}} \mathbb{1}\right] \geq 2 \mathbb{P}\left(\varsigma_{\mathfrak{g}}\left(\mathbb{S}_{\beta}\right)=0, \phi\left(\mathbb{S}_{\beta}\right)=0\right)=2 e^{-\beta(2 h+4 \lambda)} .
$$

\section{References}

1. Aizenman, M.: Geometric analysis of $\varphi^{4}$ fields and Ising models. I, II. Commun. Math. Phys. 86, 1-48 (1982) 
2. Aizenman, M., Chayes, J.T., Chayes, L., Newman, C.M.: Discontinuity of the magnetization in onedimensional $1 /|x-y|^{2}$ Ising and Potts models. J. Statist. Phys. 50(1-2), 1-40 (1988)

3. Aizenman, M., Fernández, R.: On the critical behavior of the magnetization in high-dimensional Ising models. J. Statist. Phys. 44, 393-454 (1986)

4. Aizenman, M., Klein, A., Newman, C.: Percolation methods for disordered quantum Ising models. In: Kotecky, R. (ed.) Phase Transitions: Mathematics, Physics, Biology, ..., pp. 1-26. World Scientific, Singapore (1993)

5. Aizenman, M., Nachtergaele, B.: Geometric aspects of quantum spin states. Commun. Math. Phys. 164, 17-63 (1994)

6. Baldi, P.: Large deviations and stochastic homogenization. Ann. Mat. Pura Appl. 151(4), 161-177 (1988)

7. Campanino, M., Klein, A., Perez, J.F.: Localization in the ground state of the Ising model with a random transverse field. Commun. Math. Phys. 135, 499-515 (1991)

8. Crawford, N., Ioffe, D.: On a switching lemma for quantum Ising models in transverse field, preprint (2008)

9. Dorlas, T.C.: Probabilistic derivation of a noncommutative version of Varadhan's theorem, unpublished, June 2002. http://www.stp.dias.ie/ dorlas/tony_index2.html

10. Fannes, M., Spohn, H., Verbeure, A.: Equilibrium states for mean field models. J. Math. Phys. 21, 355360 (1980)

11. Fröhlich, J., Israel, R., Lieb, E.H., Simon, B.: Phase transitions and reflection positivity. I. General theory and long-range lattice models. Commun. Math. Phys. 62(1), 1-34 (1978)

12. Grimmett, G.: Space-time percolation. Preprint, arXiv:0705.0506v1 [math.PR]

13. Guerra, F.: Broken replica symmetry bounds in the mean field spin glass model. Commun. Math. Phys. 233(1), 1-12 (2003)

14. Ioffe, D.: Stochastic geometry of classical and quantum Ising models. In: Proceedings of the 5th Prague Summer School, LNM. Springer, Berlin (2008)

15. Ioffe, D., Levit, A.: Long range order and giant components of quantum random graphs. Mark. Proc. Rel. Fields 13(3), 469-492 (2007)

16. Mézard, M., Parisi, G., Virasoro, M.A.: Spin glass theory and beyond. In: World Scientific Lecture Notes in Physics, vol. 9. World Scientific Publishing, Teaneck (1987) (preprint)

17. Nachtergaele, B.: Quasi-state decompositions for quantum spin systems in Probability Theory and Mathematical Statistics. In: Grigelionis, B., et al. (eds.) Proceedings of the 6th Vilnius Conference, pp. 565590. VSP/TEV, Utrecht, Tokyo, Vilnius (1994)

18. Nachtergaele, B.: A stochastic geometric approach to quantum spin systems. In: Probability and Phase transition, Cambridge, 1993. NATO Adv. Sci. Inst. Ser. C Math. Phys. Sci., vol. 420, pp. 237-246. Kluwer Acad. Publ., Dordrecht (1994)

19. Parisi, G.: Field theory, disorder and simulations. In: World Scientific Lecture Notes in Physics, vol. 49, World Scientific, River Edge (1992)

20. Talagrand, M.: Spin Glasses: A Challenge for Mathematicians. Cavity and Mean Field Models. A Series of Modern Surveys in Mathematics, vol. 46. Springer, Berlin (2003)

21. Talagrand, M.: The Parisi formula. Ann. Math. (2) 163(1), 221-263 (2006)

22. Toland, J.F.: A duality principle for non-convex optimization in the calculus of variations, F.M.R.I. (University of Essex), Arch. Rational Mech. Analysis (1979) 